\title{
Design of Low Pass Microwave Filter using Impulse Invariant Transform
}

\author{
Pradeep Kumar \\ Department of Electronics \& Communication \\ ABES Institute of Technology Ghaziabad, India
}

\begin{abstract}
This paper introduces a new approach to design low pass microwave filter using impulse invariant transform. In this approach, the analog specifications of desired low pass filter is transformed into the digital specification and then apply an optimization technique (DSP based) to get an approximate transfer function in digital domain. Further, the transfer function in continuous time domain is obtained by applying inverse impulse invariant technique. Now the lumped element circuit is obtained by using a classical network synthesis technique on the transfer function in analog domain. The theoretical result of low pass microwave filter is also verified on ADS Simulation tool.
\end{abstract}

\section{Keywords}

Impulse invariant transform, Low-pass filter, Microwave Filter, Quasi-Newton algorithm.

\section{INTRODUCTION}

Microwave filter is a two-port network used to control the frequency response at a certain point in a microwave system by providing transmission at frequencies within the pass band of the filter and the attenuation in the stop band of the filter. The microwave filter has been limited to classical function such as Butterworth, Chebyshev etc [1], [2]. The classical microwave filter design is limited to the image-parameter method or the insertion-loss method provides the lumpedelement circuit .The lumped element to transmission line section converted by Richard's transformation and Kuroda identities used to separate filter elements [3]. Today, most of the microwave filter designs are obtained by using the sophisticated computer-aided design (CAD) packages based on the insertion loss method. Now a day's microwave filters are also designed and implemented using digital signal processing technique [4]-[8].There are several techniques for design of microwave filter with arbitrary response[12].In this paper, a low pass microwave filter has been designed using impulse invariant transform and Quasi-Newton optimization algorithm. The theoretical result of low pass microwave filter is verified on ADS Simulation tool .Suhash C. Dutta Roy [12], the author give dual and triple pass band transformation for prototype low pass filter. A. Mohan, S. Singh, and A. Biswas [14], also give the transformation for low pass to multiple pass band filters.

\section{PROPOSED APPROACH TO DESIGN \\ MICROWAVE FILTER}

In this paper proposed design a low pass filter using impulse invariant transform, the various step for filter design is represented in block diagram as in Fig.1. The first step decide the analog specification of filter, apply impulse invariant transform convert into digital specification.With the help of quasi-Newton optimization method give the digital domain transfer function[9]. This transfer function poles and zeros are mapped into analog domain. After analog transfer function is synthesis by the recurrent-continuant method of transfer function synthesis only for low pass filter synthesis [10].The circuit component synthesis inductors and capacitors valid only 1-ohm termination of source and load impedance, and then circuit component demoralize at 50 -ohm termination or any impedance termination. After this desired microwave low pass is obtain.

\subsection{Analog domain to digital domain}

The desired microwave filter has analog specification, and the digital filter is obtained by the uniformly sampling the impulse response of the equivalent analog filter [11]. The transformation technique as follows

$$
z=e^{s T}
$$

The mapping of $j \Omega$-axis is mapped into the unit circle in the Z-plane shown in Fig.2.This mapping is valid for only band limited signal. The relationship between analog frequency and digital frequency is linear . The mapping $\omega=\Omega \mathrm{T}$ implies the interval $-\pi / \mathrm{T} \leq \Omega \leq+\pi / \mathrm{T}$ maps into the corresponding values of $-\pi \leq \omega \leq+\pi$.

\subsection{Digital Design}

There are several techniques to design a digital filter. An alternative approach for the solution of the approximation problem in digital filters is through the application of optimization methods. In these methods, a discrete-time transfer function is assumed and an error function is formulated on the basis of some desired amplitude and phaseResponse. A norm of the error function is then minimized with respect to the transfer-function coefficients. As the value of the norm approaches zero, the resulting amplitude or phase response approaches the desired amplitude or phase response. These methods are iterative and, as a result, they usually involve a large amount of computation. These methods are iterative and, as a result, they usually involve a large amount of computation. The Algorithm provides some parameter that allows us to control the design process with higher flexibility. The most relevant ones are the filter order, the weighting vector and the range of values for. As a result, the poles and zeros of the digital filters are obtained [9].The digital filter magnitude response is shown in Fig.2. 

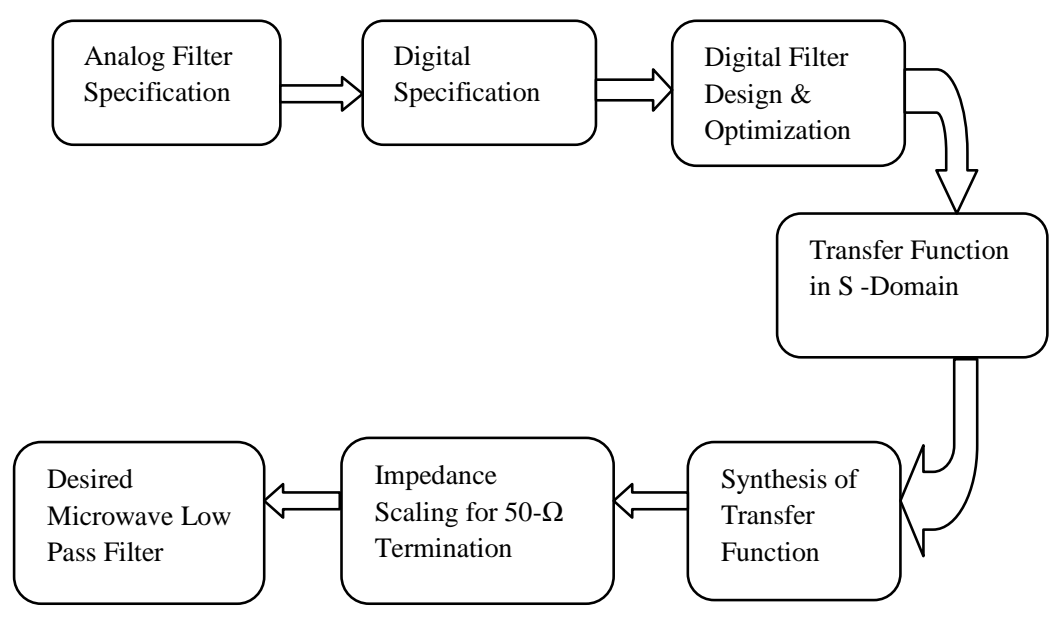

Fig1: Block diagram of digital design technique for low pass microwave filter

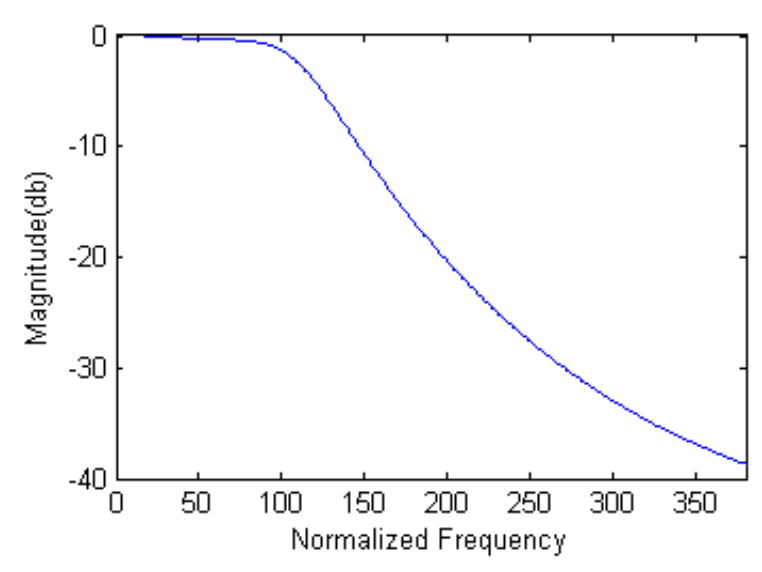

Fig 2: Digital Filter Magnitude Response

\subsection{Digital to analog conversion}

The impulse-invariant method of IIR digital filter design from a given analog filter is useful both in filter design and especially in discrete-time simulation of continuous-time systems. In the practical use of the method, it is clearly desirable that the code used in its implementation be valid for the general case involving both single- and multiple-order poles (e.g., a common application situation being a simulated plant having multiple poles at the origin).The mapping property for impulse invariant is shown in Fig.3. The analog filter magnitude response is shown in Fig.4.
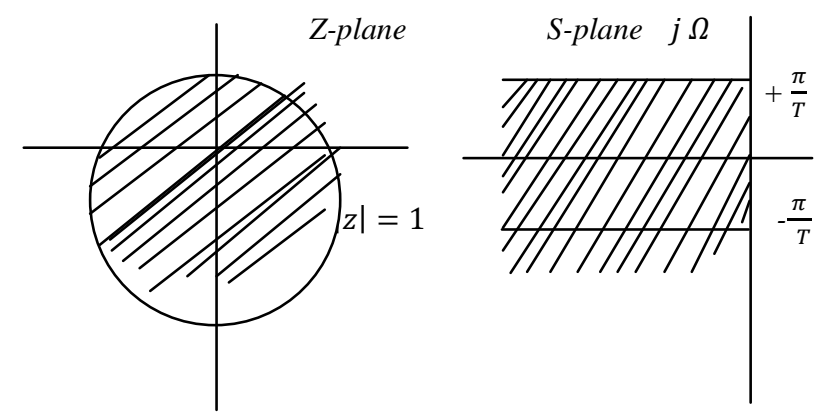

Fig. 3: Mapping between z-plane and s-plane

\section{SYNTHESIS OF MICROWAVE}

\section{FILTER}

The application of inverse impulse invariance results in the poles and zeros of analog filters. The transfer function in analog domain again changes as $e_{i n}(s) / e_{o}(s)$. This polynomial in $s$ with constant coefficients. The 5th-order lowpass network, where the polynomial is

$$
\frac{e_{\text {in }}}{e_{o}}=p_{5} s^{5}+p_{4} s^{4}+p_{3} s^{3}+p_{2} s^{2}+p_{1} s+1
$$

The numerical coefficients of (2) will completely determine a set of values for the resistors, inductors and capacitors which make up the synthesized circuit. Further, it is known that the network is normally analyzed by writing a set of equations which are usually solved by determinants as a ratio, and that the resulting voltage transfer function can even be written easily by determinants manipulation in a special form called a continuant [10].

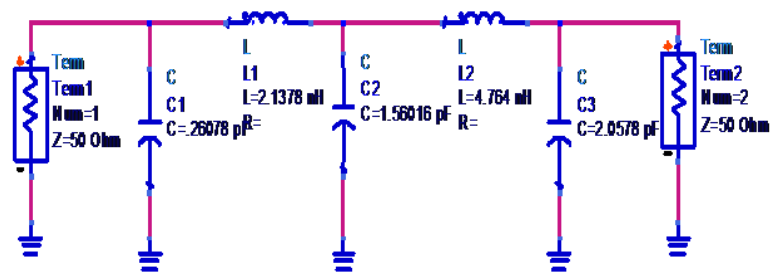

Fig 5: Ladder Network for Lumped-Element

This network synthesis has been developed, which links the coefficients of a given transfer polynomial directly to the network element values by means of the special recurrentcontinuant determinant transformation. This method of synthesis is limited only low pass transfer function. After synthesis apply suitable frequency transformation this convert like, band-pass, high-pass, band reject etc.

\section{EXAMPLE}

In this section proposed low pass filter 5 th order .The filter specification in analog domain, pass band of filter is dc to 2.8 . $\mathrm{GHz}$. The sampling frequency is $20 \mathrm{GHz}$. The transfer function in digital domain is as follows:

$$
\frac{0.06495}{\left(z^{5}-2.541 z^{4}+2.76 z^{3}-1.52 z^{2}+0.4072 z-0.04092\right)}
$$

The design approach has been discussed in section 3 , and its Frequency response is shown in Fig.3. After apply the inverse impulse invariant transform as explained in above section 2.1 .C, the digital poles and zeros obtained are mapped in to the analog domain. The transfer function in analog domain is as follows

$$
\frac{2.344 e^{51}}{\left(s^{5}+7.6 e^{10} s^{4}+2.3 e^{21} s^{3}+4.1 e^{31} s^{2}+4.5 e^{41} s+2.3 e^{51}\right)}(4)
$$

Where $\mathrm{e}=10$, from the analog poles and zeros obtained in the mapping process, a lumped element ladder network is obtained in section 3. The capacitances values are $\mathrm{C} 1=0.26078 \mathrm{pF}, \mathrm{C} 2=1.56016 \mathrm{pF}, \mathrm{C} 3=2.0578 \mathrm{pF}$ and the values of inductances $\mathrm{L} 1=2.1738 \mathrm{n} \mathrm{H}, \mathrm{L} 2=4.764 \mathrm{n} \mathrm{H}$ in figure5. The magnitude response of filter on Advanced Design System is given below in Fig. 6 . 


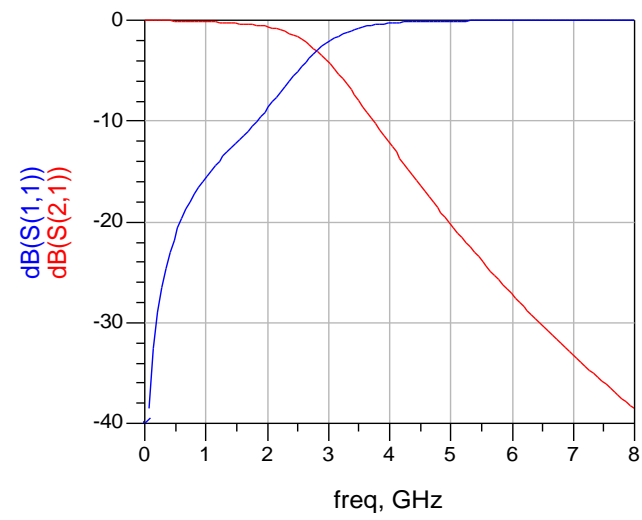

Fig.6: Simulation of Filter Magnitude Response on ADS

\section{CONCLUSION}

In this paper proposed digital design technique for low pass microwave filter can be generalized for multiple pass-band filters. This digital design technique can be extended for dual and triple pass band filter by using frequency transformation. The lumped circuit equivalent of low pass filter can be converted into lumped dual pass band filter and using Richards and Kuroda identity converted into distributed component for hardware realization. This digital technique and circuit synthesis is very simple and efficient for microwave designer. The simulation response of low pass filter is verified on Advanced Design System (ADS) and MATLAB.

\section{ACKNOWLEDGMENTS}

The author is highly thankful to D Upadhyay, A.P at Division of Electronics \& Communication Netaji Subhas Institute of Technology ,Dwarka, New Delhi, India (Delhi University) who give the valuable suggestion and good guidance for this research work specially for microwave filters.

\section{REFERENCES}

[1] W.-K. Chen, The Circuits and Filters Handbook. Orlando, FL: CRC, 1995.

[2] G.Matthaei,L.Young, and E.M.T.Jones , Microwave Filters, Impedance-Matching Networks, and Coupling Structures. Norwell, MA: Artech House, 1980.

[3] David M. Pozar, Microwave Engineering, John Wiley \& Sons, Inc., New York, 1998.

[4] Da-Chiang and Ching-Wen Hsue, "Design and Implementation of filters using transfer in the $\mathrm{Z}$ Domain," IEEE Trans. Microw. Theory Tech., vol.49, no.5, May 2001.
[5] G. Macchiarella and S. Tamiazzo," Design techniques for dual-passband filters", IEEE Trans. Microw. Theroy Tech., vol. 53, no. 11, pp.3265- 3271, Nov. 2005.

[6] S. Bila,D. Baillargeat,S. Verdeyme, M. Aubourg, P. Guillon, F. Seyfert, J. Grimm, L. Baratchart, C. Zanchi, and J.Sombrin, "Direct electromagnetic optimization of microwave filters ,"IEEE Microw. Mag., vol. 2, no. 1, pp. 46-51,Mar. 2001.

[7] C.Charalambous, "A unified review of optimization," IEEE Trans. Microw. Theory Tech., vol. MTT-22, no. 3, pp. 289-300, Mar. 1974.

[8] Israel Arnedo, A. Lujambio, T. Lopetegi, Member, "Design of Microwave Filters With Arbitrary Frequency Response Based on Digital Methods.” IEEE Microw. Theory Tech., vol. 17,no. 9, pp.634-636, Sept. 2007.

[9] A. Antoniou, Digital Signal Processing. New York: McGraw-Hill, 2005.

[10] Holbrook, J.G, "The recurrent-continuant method of transfer function synthesis." The Radio and Electronic Engineer, vol.38, no. 2, pp. 73-81, August 1969.

[11] Thomas J.Cavicchi, "Impulse Invariance and MultipleOrder Poles.” IEEE Trans. Signal process., vol. 44, no. 9, pp.2344-2347, Sept.1996.

[12] Herbert L. Thal, "Design of Microwave Filter with Arbitrary Response” John Wiley and Sons ,Inc. 1997

[13] Suhash C. Dutta Roy, "On the Design of a Multiple Passband Filter by frequency transformation." IETE Journal of Research, vol-58,Jan-Feb-2012.

[14] A. Mohan, S. Singh, and A. Biswas "Generalized Synthesis and design Of Symmetrical multiple pass band filters”, PIER B, vol-42,115-139, 2012.

\section{ABOUT AUTHOR}

Pradeep kumar was born in Bareilly Uttar Pradesh. He received the degree of B.Tech in Electronics \& Instrumentation in 2005 U P T U Lucknow , M.Tech in Signal Processing in 2012 NSIT New Delhi, Delhi University, India. $\mathrm{He}$ is Assistant Professor in Electronics \& Communication Dept. of ABES Institute of Technology Ghaziabad. His field of research area are Circuit theory, Digital Signal Processing, Analog Signal processing and Microwave filter design. 